\title{
PREVENCIÓN DE LAS PRINCIPALES LESIONES EN LA DANZA Y MECANISMOS DE PRODUCCIÓN
}

\author{
Alicia Corrales Valero \\ María Mena Milán \\ Juan José García Jaén \\ Remedios López-Liria \\ Universidad de Almería \\ (alicovalero@gmail.com) \\ https://doi.org/10.17060/ijodaep.2017.n1.v2.936
}

Fecha de Recepción: 19 Enero 2017

Fecha de Admisión: 1 Abril 2017

\section{RESUMEN}

La danza clásica y la española es una forma de arte que requiere de un gran esfuerzo por parte de quienes lo practican, ya que utiliza movimientos muy extremos del cuerpo.

El objetivo de este trabajo ha sido conocer a través de una revisión bibliográfica de la literatura existente, las distintas técnicas y abordajes que se pueden aplicar para prevenir las lesiones de tipo mecánico que aparecen con más frecuencia en este arte, como son los esguinces, roturas musculares, fracturas y lesiones tendinosas producidas sobre todo en tobillos, rodillas, pies y espalda.

La información fue obtenida de bases de datos científicas, como PubMed, PeDro o Elsevier y otras fuentes secundarias. Las palabras claves tanto en español como en inglés han sido: "baile", "lesión”, "prevención”, "prevalencia”, "flamenco", "danza”, "dance”, "injury”, "prevention”, "prevalence", "common", "frequent", "lession”, "flamenco" y "ballet".

La mayor parte de las lesiones se producen en los miembros inferiores y están relacionadas con la sobrecarga 0 debilidad muscular, más que a traumatismos. Estas lesiones también suelen estar provocadas por una falta de técnica o la aplicación errónea de la misma, o en ocasiones por mal acondicionamiento de la sala donde se practica.

Palabras clave: Fisioterapia, Psicología, Danza, Lesiones, Prevención.

\section{ABSTRACT}

Common dancing injuries prevention and production mechanisms

Dance is a form of art that requires of a big effort from those who practise it, as it makes use of very extreme body movements.

The aim of this work is to get to know, through a bibliographic revision of the existing literature 


\section{PREVENCIÓN DE LAS PRINCIPALES LESIONES EN LA DANZA Y MECANISMOS DE PRODUCCIÓN}

in this field, the different techniques and approaches that can be applied to prevent the most frequent mechanic injuries in dance, such as sprains, muscular breaks, fractures and sinew injuries mainly produced in ankles, knees, feet and back.

The information was collected from scientific databases, such as PubMed, PeDro, Elsevier and other secondary sources. The keywords both in Spanish and English were: "baile", "lesión", "prevención", "prevalencia", "flamenco", "danza”, "dance”, "injury”, "prevention”, "prevalence”, "common", "frequent", "lession", "flamenco" and "ballet".

The majority of injuries occur in the lower extremities and are often related to muscular overload or weakness, rather than traumatisms. These injuries are commonly produced by a lack of technique or a wrong application of it, or even sometimes by the bad fitting-out of the room where the activity is being practised.

Keywords: dance, injuries, physiotherapy, prevention, exercises, psychology

\section{INTRODUCCIÓN}

La danza es una forma de arte que requiere de un gran esfuerzo por parte de quienes lo practican, ya que utiliza movimientos muy extremos del cuerpo. Podemos distinguir dos tipos de danza, la clásica y la española.

Los bailarines suelen comenzar a temprana edad, sobre los 8 años y su dedicación de forma profesional suele acabar alrededor de los 30 , debido a los componentes físicos de esta disciplina (Wainwirght et al., 2005).

El entrenamiento requiere muchas horas, ya que se centra en conseguir, además de una buena técnica, una adecuada flexibilidad, fuerza, resistencia y velocidad (Gómez Martínez, 2012; Steinberg et al., 2013).

Se espera de los bailarines que posean una enorme flexibilidad, en muchos casos heredada, en otras adquirida (Howse, 2011), pero en ambos casos, ésta aumenta con el entrenamiento. Pero para que esta flexibilidad no se convierta en un problema, es muy importante también fortalecer la musculatura.

Al ser un trabajo físico, con muchas horas de entrenamiento, ocurren muchas lesiones. Los principales factores son la falta o mala aplicación de la técnica (Howse, 2011) y la debilidad muscular, pero encontramos más causas como por ejemplo, las herramientas que se usan al bailar (Castilla-Cubero \& Jiménez-Sarmiento, 2011).

En el caso de la danza clásica o ballet, puede deberse al uso de las zapatillas de punta, donde se emplean los músculos intrínsecos del pie y el complejo sural (Howse, 2011). Unas zapatillas en mal estado, muy usadas, demasiado pequeñas o demasiado grandes, pueden llevar al bailarín a lesionarse.

En el caso de la danza española, además del trabajo que también se realiza con zapatillas de media punta 0 alpargatas, se usan castañuelas, mantón o bata de cola entre otros, junto al zapato de tacón, que es el que más lesiones produce por el elevado número de horas que el bailarín lo tiene puesto, y por la gran fuerza que ejerce en cada zapateado (Echegoven et al., 2013).

Otra causa puede ser cuando el docente no repara en las limitaciones físicas o funcionales de cada bailarín forzando una técnica defectuosa para el mismo, o no corrige los fallos en la realización inadecuada y lesiva de la técnica. Además, es muy importante que se insista en realizar un calentamiento previo a la actividad, que incluya ejercicios de activación, movilidad y estiramientos, así como realizar siempre estiramientos al final de la sesión (Howse, 2011).

También existen motivos ambientales que repercuten en la aparición de lesiones como son una temperatura inadecuada de la sala. El suelo es un factor muy importante, ya que en ocasiones se 
utilizan materiales no elásticos y existe una falta de amortiguación, provocando lesiones podales y lumbares, sobre todo al realizar saltos o zapateados, provocando incluso fracturas por sobrecarga (Albisetti et al., 2010).

Otros factores internos dependientes del individuo, son la atrofia generalizada (tras una lesión 0 tiempo de reposo), mala nutrición, hábitos tóxicos, una capacidad respiratoria disminuida (asma), el aumento del peso corporal en el bailarín o por efectos psicológicos (esta disciplina requiere una gran concentración para no producir errores en la técnica que lleven al bailarín a la lesión) (Howse, 2011).

La justificación de este trabajo se debe a que existe muy poca literatura e investigación sobre el tema a pesar de la importancia y el interés que suscita desde el punto de vista sanitario y de los bailarines.

\section{OBJETIVOS}

Describir las lesiones más frecuentes que existen en la danza clásica, en la danza española y el baile flamenco a través de la información adquirida tras una revisión bibliográfica. Y señalar cómo prevenirlas desde el punto de vista de la fisioterapia.

\section{METOTOLOGÍA}

La información ha sido obtenida de una exhaustiva búsqueda bibliográfica procedente de varias bases de datos científicas, como PubMed, PeDro o Elsevier y de otras fuentes secundarias, como diferentes libros de anatomía, fisioterapia, biomecánica y danza, debido a la escasez de investigación científica en relación al tema. Las palabras claves tanto en español como en inglés han sido: “baile”, "lesión”, "prevención”, "prevalencia”, "flamenco", “danza”, "dance”, “injury”, "prevention”, "prevalence”, "common”, "frequent”, "lession”, "flamenco" y "ballet”. Básicamente, todas las investigaciones halladas fueron en inglés.

\section{RESULTADOS}

\section{A) LESIONES MÁS PREVALENTES}

En la danza clásica o ballet, encontramos que las lesiones más frecuentes se producen en: tobiIlo $(29 \%)$, pie $(18 \%)$, rodilla $(10.5 \%)$, cadera $(9 \%)$, columna lumbar $(8 \%)$, columna cervico-dorsal (8\%), MMII (6.5\%), hombro (6\%) (Bowling, 1989).

En el caso de la danza española o el flamenco, la prevalencia cambia. Esto es debido al diferente uso de materiales y a los distintos movimientos que se realizan, ya que se usa otra técnica distinta. En este caso, se producen en: tobillo (23\%), espalda (20\%), pie (17\%), cuello ( $7 \%$ ), y otras (3\%) (Bowling, 1989).

B) PREVENCIÓN DE LAS LESIONES MÁS FRECUENTES EN LA DANZA CLÁSICA

Las lesiones que se producen con más frecuencia en la danza clásica son: el esguince de tobiIlo, la fractura por estrés del $2^{0}$ y $3^{\circ}$ metatarsianos, la tendinopatía del tendón de Aquiles, la uña encarnada y las lesiones musculares.

\section{B.1. ESGUINCE DE TOBILLO.}

Supone el estiramiento de las fibras del ligamento en más de un $5 \%$ de su capacidad funcional. Es una lesión microscópica, donde los síntomas aparecen al cabo de 2-6 horas después, siendo dolor, inflamación, espasmo e incapacidad funcional relativa. Precisa reposo (Coltart et al., 1951; Robertson, 1967).

El "paso" donde más suele producirse esta lesión es durante el "relevé". Un relevé es lo que comúnmente se conoce como "ponerse de puntillas", pero al máximo, es decir, una máxima flexión plantar y, generalmente, con el o los miembros inferiores en completa extensión. Esta flexión plan- 


\section{PREVENCIÓN DE LAS PRINCIPALES LESIONES EN LA DANZA Y MECANISMOS DE PRODUCCIÓN}

tar debe realizarse de forma progresiva. Con zapatillas de media punta, los dedos de los pies quedarán en flexión dorsal, apoyados en el suelo. En el caso de las zapatillas de punta, la flexión plantar será completa, también con los dedos.

Lo más frecuente, es que se produzca durante la reproducción de algún giro o pirouette, que deben hacerse en relevé (figura 1) ya sea a media punta o a punta, o en la recepción o caída de algún salto, donde se realiza una triple flexión de miembros inferiores. Ésta debe ser amortiguada y elástica, ya que si se cae de forma brusca, se provoca lesión (figura 2).

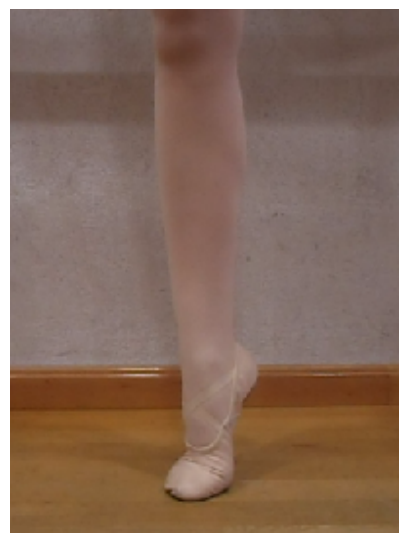

Figura 1. Correcta posición de relevé

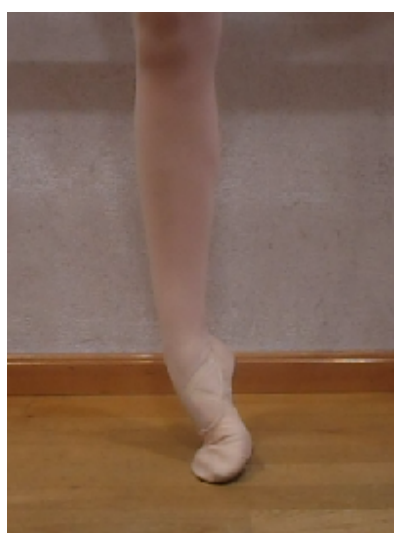

Figura 2. Posible esguince de tobillo

El motivo más común de lesión, suele ser una debilidad de los músculos del tobillo (Tixa, 2006).

Para prevenir esta lesión, es necesario concienciar al docente y al bailarín en usar la técnica correctamente, y si no fuera suficiente, se elaborará una tabla de ejercicios para que fortalezcan los músculos que más se afectan, intentando evitar estas lesiones.

\section{B.2. FRACTURA POR ESTRÉS DEL II Y III METATARSIANOS}

Una fractura por estrés es una interrupción ósea que resulta de una fuerza repetida menor que la fuerza requerida para fracturar el hueso con una sola carga. Las fracturas por estrés en el segundo y en el tercer metatarsiano son muy comunes en los bailarines de ballet (Albisetti et al., 2010). La fractura suele ser en medio de la diáfisis o en la base. Uno de los factores que lo causa es la discrepancia en la longitud de los metatarsianos; también los dedos del pie largos o los antepiés débiles (Howse, 2011).

Los pasos donde más comúnmente se producen este tipo de lesiones, son en los que se usan las zapatillas de punta. Generalmente, son échappée y relevé, pasos donde se produce una carga repentina sobre los dedos. El échappeé es un relevé que se realiza de forma brusca y rápida. Si se realiza de manera incorrecta con un pequeño salto o rebote a la llegada del relevé con las piernas en extensión completa, pueden producir la lesión.

A continuación, las fases de un salto batido desde 5ํㅜ posición (figuras 3,4 y 5): 


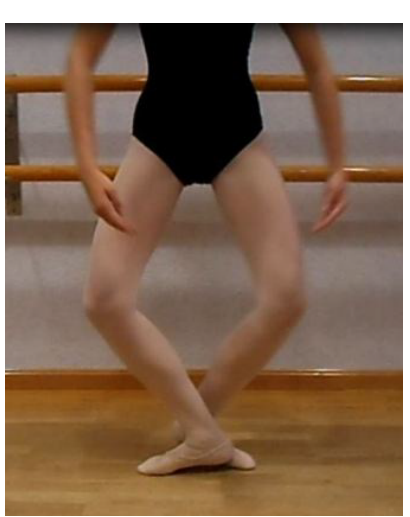

Figura 3. Despegue

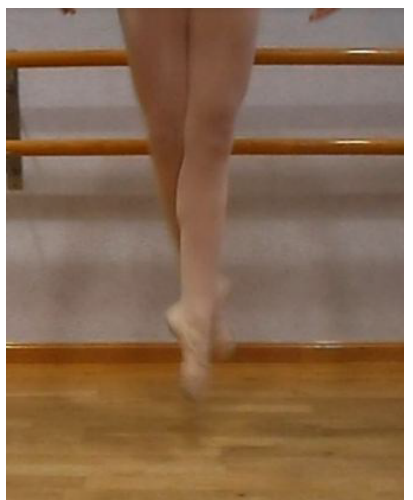

Figura 4. Fase de vuelo

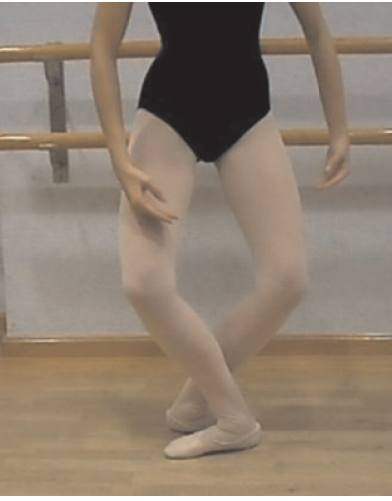

Figura 5. Recepción

Para prevenir las posibles lesiones, es necesario fortalecer los músculos intrínsecos del pie (Tixa, 2006) además de no hacer sobreuso de la articulación y en el caso de los échappées evitar ese rebote.

\section{B.3. TENDINOPATÍA EN EL TENDÓN DE AQUILES}

El tendón de Aquiles es el tendón más grueso y potente del organismo humano, se inserta en el hueso calcáneo del pie y permite que en cada paso la articulación del tobillo se flexione hacia la planta del pie. En los saltos el tendón absorbe el peso corporal, tolerando una elevada tensión de tracción excéntrica e impidiendo que el músculo gemelo de la pierna se sobredistienda y se comprima el calcáneo (Freese, 2006).

Al igual que ocurre con otras estructuras, los tendones reaccionan a sobrecargas repentinas, a la alineación defectuosa de las articulaciones y a fuerzas desequilibradas que generan dolor, en cuyo caso se produce un engrosamiento del tendón y una alteración de su eficacia. En el caso del tendón de Aquiles, éste no cuenta con una vaina, por lo que las patologías inflamatorias en este área afectan el tejido fibroso laxo que rodea el tendón (Kulig et al., 2011). La causa puede ser un uso excesivo, que acaba provocando dolor en el tendón. Se agrava por la hiperextensión de las rodillas o por bailar con el peso demasiado atrás.

También puede ser causada por una falta de trabajo de los tres cuartos de pointé (figura 6), e incluso por el uso de una zapatilla no adecuada.

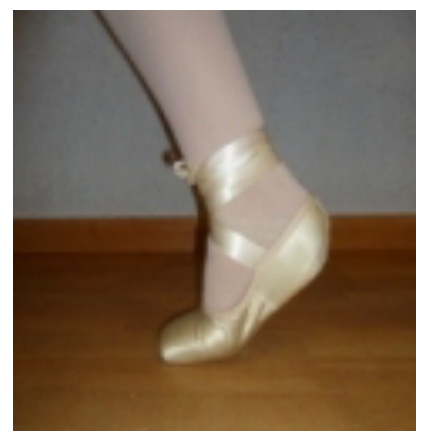

Figura 6. Trabajo de los tres cuartos de pointé 


\section{PREVENCIÓN DE LAS PRINCIPALES LESIONES EN LA DANZA Y MECANISMOS DE PRODUCCIÓN}

Para prevenir esta patología se precisa no hiperextender rodillas, flexionándolas mínimamente en el caso de que el bailarín tenga hiperextensión y fortaleciendo cuádriceps; además es importante cuidar el reparto del peso.

\section{B.4. UÑA ENCARNADA}

Se produce cuando la uña del primer dedo del pie se tiene muy curvada en los laterales y se hunde en la carne verticalmente. La zapatilla de punta, por la zona de la punta es rígida, ya que tiene que llegar a soportar todo el peso del cuerpo, y está fabricada generalmente con madera o tela prensada. En el caso de que quede demasiado estrecha, provocará que el pie quede demasiado aplastado, provocando esta lesión (Howse, 2011). Para prevenirlas, se deben usar unas zapatillas de punta que se adapten a cada pie, y tener una buena higiene (llevar las uñas recortadas).

B.5. LESIÓN MUSCULAR

Las lesiones de los músculos gastrocnemios y sóleo son las más habituales. Se suele producir una distensión aguda del gastrocnemio durante los despegues para un salto 0 tras un sobreestiramiento excéntrico repentino. Se siente un desgarro o dolor punzante, agudo y súbito en el vientre del músculo. Una distensión de primer grado es aguda pero el bailarín puede continuar con su entrenamiento. En una distensión de segundo grado, el bailarín no puede continuar y la lesión tarda hasta tres semanas en recuperarse. Una distensión de tercer grado supone una rotura con dolor intenso y es posible que requiera cirugía o llevar una venda enyesada.

Lo fundamental en estos casos para prevenir la lesión, es haber realizado un correcto calentamiento antes de empezar la actividad. El calentamiento debe estar formado por ejercicios de fuerza y de elasticidad. También es esencial no sobrepasar los límites físicos de cada uno. Aunque para conseguir tener más flexibilidad es necesario la realización de diversos estiramientos (tanto pasivos 0 activos, como concéntricos o excéntricos) no debemos estirar más de lo que nuestro cuerpo permite (Howse, 2011).

C) LESIONES MÁS FRECUENTES EN LA DANZA ESPAÑOLA Y EL BAILE FLAMENCO

Algunas de las lesiones que se producen con mayor frecuencia en estas disciplinas son: las fracturas por sobrecarga de las vértebras lumbares, sesamoiditis, hallux valgus, fascitis plantar, tendinopatía rotuliana.

\section{C.1. FRACTURA POR SOBRECARGA DE LAS VÉRTEBRAS LUMBARES}

En el caso de las vértebras lumbares, se produce sobre todo a nivel de L4 y L5, siendo más frecuentes las fracturas bilaterales. Si no se detecta pronto la fractura, se puede ensanchar gradualmente y separarse, de modo que el cuerpo de la vértebra y toda la columna por encima de ese nivel se desplacen lentamente hacia delante. Una vez se ha iniciado este proceso, no hay posibilidad de que la fractura consolide. La causa más importante es la falta de estabilización y activación de los músculos abdominales profundos. La mayor tensión se localiza en el punto donde la columna lumbar se inserta en la pelvis en la unión lumbosacra L5/S1 o en L4/L5.

Normalmente este tipo de lesiones suele producirse en saltos o echappés, pero también influye muchas horas de entrenamiento con zapateados, ya que la vibración se transmite por toda la columna. En el zapateado, podemos usar la media punta (golpeando sólo con los dedos), la planta (golpeando con la planta entera del pie), del tacón (golpe con el tacón del zapato) 0 con la punta (golpeando con la punta de los dedos). Estos cuatro elementos, se van combinando y debe hacerse con fuerza para que suene, aunque dependiendo del montaje coreográfico, su intensidad deberá ir variando (Howse, 2011). El tratamiento inmediato sería reposo, para que remitan los síntomas. 


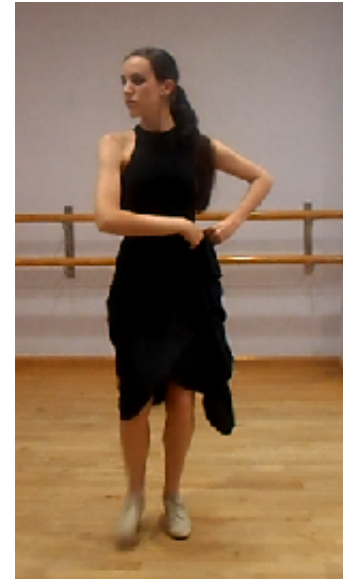

Figura 7. Fase del zapateado

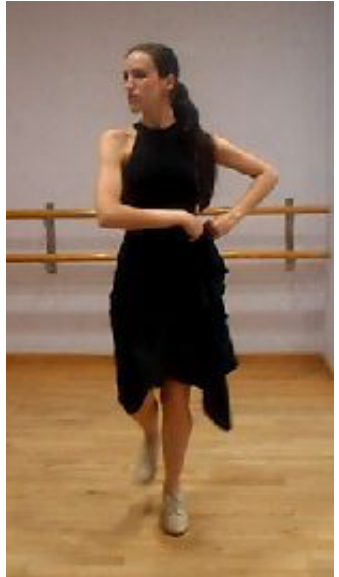

Figura 8. Fase del zapateado /I

Para prevenir este tipo de lesiones es importante fortalecer la musculatura abdominal profunda y sobre todo evitar una postura lordótica que genere más rotación externa de las caderas y la rotación externa en general.

\section{C.2. SESAMOIDITIS}

Cuando en los huesos sesamoideos se producen magulladuras, inflamación o dolor, nos encontramos ante una sesamoiditis. Suele presentar dolor local y edema. La sensibilidad dolorosa al tacto se localiza bajo la cabeza del primer metatarsiano. Generalmente es provocada por un traumatismo directo, por un mal aterrizaje o por el trabajo en una superficie dura, unos zapatos en malas condiciones y también aparece cuando existe un repetitivo zapateado donde se da con la punta en el suelo.

El tratamiento suele ser lento y podemos emplear hielo y ultrasonidos. La prevención de este tipo de lesiones es complicada, ya que no existe nada concreto que podamos evitar. Simplemente, usar una buena técnica y una sala bien acondicionada.

\section{C.3. HALLUX VALGUS}

El hallux valgus, popularmente conocido como "juanete" es una deformidad, generalmente adquirida, que afecta al quinto metatarsiano y quinto dedo del pie (Castillo et al., 2013). Se asocia, normalmente, a una marcada desviación medial del quinto dedo y a una desviación lateral del $\mathrm{V}$ metatarsiano; así como a una inflamación de la bursa y tejidos blandos de la quinta articulación metatarsofalángica, dando lugar a estadios dolorosos. El origen del Juanete de Sastre es multifactorial: o bien sea un metatarso primo varo congénito que produce hallux valgus secundario, apareciendo después un hallux valgus por el uso de un calzado deficiente; 0 si el niño/a empieza a ponerse de puntillas demasiado pronto, cuando los pies aún no son lo bastante fuertes. También encontramos entre las causas principales, un rodamiento muy prolongado o debilidad en los músculos intrínsecos del antepie (Tixa, 2006).

El músculo aductor del primer dedo es uno de los responsables de que se mantenga el hallux valgus. Además la pronación anormal de la articulación subastragalina, la posición en varo del antepié 0 del retropié no compensada, o la deformidad congénita en flexión-plantar o dorsi-flexión del quinto radio también pueden llegar a causar esta lesión. 


\section{PREVENCIÓN DE LAS PRINCIPALES LESIONES EN LA DANZA Y MECANISMOS DE PRODUCCIÓN}

Dentro del tratamiento, podemos encontrar la opción de la cirugía, pero no es recomendable en todos los casos. En el caso de tratamiento conservador, se recomendará el uso de un anillo almohadillado de fieltro que alivie la presión local sobre el juanete. Podría prevenirse encontrando un zapato que se adapte al pie del bailarín y no lo fuerce; y sobre todo, fortaleciendo la musculatura intrínseca del pie.

\section{C.4. FASCITIS PLANTAR}

La fascitis plantar es una inflamación del tejido conectivo que sostiene a modo de cuerda el arco que forma la planta del pie. El afectado siente un dolor punzante por debajo del talón al andar o saltar normalmente (Freese, 2006). Una de las causas es una irritación crónica por una pronación excesiva, que provoca microdesgarros en la fascia. Suele presentarse en pies muy débiles y se agrava cuando se llevan zapatillas demasiado cortas.

Para su tratamiento inicial, se recomienda la aplicación de hielo, ultrasonidos y la realización de ejercicios de fortalecimiento.

\section{C.5. TENDINOPATÍA ROTULIANA}

También se le llama "rodilla del saltador" y se suele localizar en la unión osteotendinosa del tendón rotuliano. Las causas que la producen pueden ser: un desequilibrio del cuádriceps entre sus componentes medial y lateral; rodamiento o rotación externa excesiva; 0 al echar el peso del cuerpo demasiado atrás. También si el tendón de Aquiles se encuentra con mucha tirantez, como ocurre durante los zapateados, el pie no logra absorber el choque, soportando la tensión el tendón rotuliano. Se basa en inflamaciones del propio tejido tendinoso o de las superficies de deslizamiento circundantes (Echegoven et al., 2013).

Para prevenirla, además de evitar todos los factores de riesgos ambientales y la realización de una buena técnica, será importante fortalecer la musculatura general de los miembros inferiores, ya que una debilidad en cualquier punto, provocará una lesión, debido a la gran fuerza y vibración generada durante un zapateado. Y muy importante, cuando se zapatee, mantener una flexión de rodillas, no exagerada, pero que permita la amortiguación.

\section{DISCUSIÓN}

En este artículo se trata de ofrecer de un modo claro y práctico cómo se producen algunas lesiones en los bailarines/as y qué factores están relacionados, de manera que pueda orientárseles a la hora de realizar una prevención de las mismas.

Se ha hallado que la mayor parte de dichas lesiones se producen en los miembros inferiores, cuestión que es debida entre otros motivos, a la propia técnica de la danza clásica.

En esta disciplina, existen numerosos pasos complicados con rápidos y repetitivos movimientos de las piernas, pasos que requieren mucha resistencia y elasticidad con elevaciones de las piernas y posiciones anatómicamente no funcionales, que repercuten y perjudican al cuerpo. Hay numerosos saltos en los que se necesita conseguir mucha elevación y además algunos se componen de baterías o battu (es decir, que baten los pies en el aire). En muchos casos, si no se eleva lo suficiente el miembro, cuando se desciende hacia el suelo es posible que se hagan daño al caer. De ahí, la especial relevancia de entrenar una suficiente potencia en las piernas para saltar a una altura recomendable, por ejemplo en este tipo de baile.

Entre las técnicas de los bailarines, también existen medianos y grandes saltos donde los desplazamientos llegan a ser de varios metros. Lo que precisa que el bailarín potencie la musculatura de los miembros inferiores de una forma exquisita, haciendo hincapié en cada uno de los músculos, para ganar en la altura del salto. Es el caso por ejemplo del grand jeté, un salto en el que las piernas llegan a abrirse por completo en el aire, en el que además de la dificultad del desplazamiento, 
el salto comienza desde una sola pierna y la recepción también será sobre una pierna soportando el peso del cuerpo además de la velocidad del movimiento.

La dificultad de este salto, entraña una gran posibilidad de producirse un esguince si la musculatura del tobillo no es potente y la rodilla también podría llegar a sufrir una lesión si no se amortigua adecuadamente la caída.

Como ya se ha ido indicando, en todos estos saltos, la planta del pie se resiente al cabo del tiempo, provocando otro tipo de lesiones como son las fracturas por estrés, las lesiones musculares 0 las tendinopatías.

Por otro lado, en el caso de la danza española, no se debe olvidar que además de los miembros inferiores que se afectan en la mayoría de los casos, también sufre mucho la columna vertebral y la espalda en general, debido al uso del zapato de tacón. Cada zapateado provoca un gran número de vibraciones que se transmiten a lo largo de todo el cuerpo, provocando desequilibrios en la anatomía, que pueden llegar a fracturar algunas vértebras, provocar sesamoiditis o incluso fascitis plantar.

Anque el flamenco sea quizás la rama más conocida de la danza española, ésta también se conforma por otras disciplinas, como son el folklore, la escuela bolera o la danza estilizada, por lo que dependiendo de cada género, el bailarín usará unas herramientas u otras (desde las zapatillas de media punta 0 las alpargatas, hasta la bata de cola o las castañuelas).

Por otro lado, la danza española es un arte considerado como de los más completos a la hora de trabajar con el aparato musculoesquelético, y por ello, también se pueden encontrar un gran número de lesiones de diferentes características. Además de la repercusión que tiene el uso del zapato de tacón, por ejemplo las castañuelas pueden llegar a provocar tendinitis en los antebrazos. También el peso de la bata de cola requiere que las piernas estén fuertes, ya que de no ser así, se realizan compensaciones con otras partes del cuerpo, influyendo erróneamente tanto en la técnica como en la columna, que podrá lesionarse.

Existe diversidad de factores ambientales que afectan a la calidad del entrenamiento en los bailarines. Es evidente que pueden existir notables diferencias en la calidad de la enseñanza según las escuelas, sus recursos, las condiciones de la sala donde se practica, las horas de entrenamiento 0 la situación personal del bailarín. Paradójicamente, en ocasiones, se observa que es peor la situación personal del bailarín en la mejor escuela, debido a la presión y la competitividad que pueda sufrir en este ámbito, por lo que no se deberían obviar ciertos factores psicológicos que afectan en la producción de las lesiones.

Respecto a las limitaciones halladas en este trabajo, ha de señalarse que los resultados sólo abordan un reducido número de artículos que se refieren específicamente a la danza. La mayoría de la documentación describe temas específicos sobre alguna lesión muy concreta o tratamiento, sin apenas tratar la prevención de las lesiones en el baile. Es preciso que se lleven a cabo estudios epidemiológicos sobre este tema a nivel nacional e internacional, debido a la magnitud de escuelas de danza que existen en todo el territorio y la relevancia que está adquiriendo esta práctica.

\section{CONCLUSIONES}

La mayor parte de las lesiones tanto en la danza clásica como en la española y el baile flamen$\mathrm{co}$, son producidas en los miembros inferiores. Y suelen estar asociadas con gran probabilidad a la sobrecarga o debilidad muscular, más que a traumatismos.

Estas lesiones están íntimamente relacionadas con la misma disciplina, que exige numerosas horas de entrenamiento, además de posturas y movimientos que en ocasiones no son ergonómicos. Entre las principales causas puede estar una falta de técnica. 


\section{PREVENCIÓN DE LAS PRINCIPALES LESIONES EN LA DANZA Y MECANISMOS DE PRODUCCIÓN}

En general, no se le ofrece la debida relevancia a los factores ambientales en la producción de estas patologías; aunque se destaca la necesidad de un buen acondicionamiento del bailarín.

\section{REFERENCIAS BIBLIOGRÁFICAS}

Albisetti W., Perugia D., De Bartolomeo 0., Tagliabue L., Camerucci E., Calori GM. (2010). Stress fractures of the base of the metatarsal bones in young trainee ballet dancers. International Orthopaedics, 34(1), 5-51.

Bowling, A.(1989). Injuries to dancers: prevalence, treatment, and perceptions of causes. British Medical Journal, 298(6675), 4-731.

Castilla-Cubero JL, Jiménez-Sarmiento AS. (2011) Aspectos generales sobre el dolor osteomuscular en el baile flamenco. Rehabilitación, 45(2), 117-121.

Castillo JM, Munuera PV, Domínguez G, Salti N, Algaba C. (2013). Prevalencia del juanete de sastre y quinto dedo adducto varo en el baile flamenco profesional. Revista Científica del Centro de Investigación Flamenco Telethusa, 6(7), 13-18.

Coltart W.D., M.B., F.R.C.S.(1951). Refresher course for general practitioners "Sprained ankle". British Medical Journal, 657-961.

Echegoyen S, Aoyama T, Rodríguez C. (2013) Zapateado Technique as an Injury Risk in Mexican Folkloric and Spanish Dance. Medical Problems of Performing Artists, 1-4.

Freese, J. (2006). Fitness terapeútico. 1st ed. Badalona: Paidotribo.

Gomez Martinez, M. (2012). Manual de anatomia y biomecanica aplicada a la danza. Murcia: DM.

Howse, J. (2011). Tecnica de la danza. Barcelona: Paidotribo.

Kulig K, Loudon JK, Popovich JM, Pollard CD, Winder BR. (2011). Dancers With Achilles Tendinopathy Demonstrate Altered Lower Extremity Takeoff kinematics. Journal of Orthopaedic and Sports Physical Therapy, 41(8), 606-613.

Robertson I. (1967). Injuries of the leg in football and ballet. Journal of the Royal Society of Medicine, 60(6), 530-532.

Steinberg N, Siev-Ner I, Peleg S, Dar G, Masharawi Y, Zeev A, Hershkovitz I. (2013). Injuries in Female Dancers Aged 8 to 16 Years. Journal of Athletic Training, 48(1), 118-123.

Tixa S. (2006). Atlas de anatomía palpatoria. 2nd ed. Barcelona: Elsevier.

Wainwright SP, Williams C, Turner BS. (2005). Fractured identities: injury and the balletic body. Health, 9(1), 49-66. 